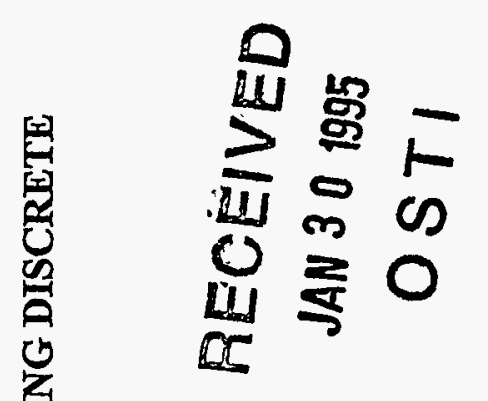

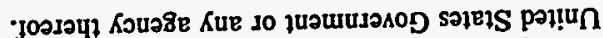

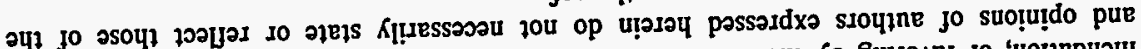

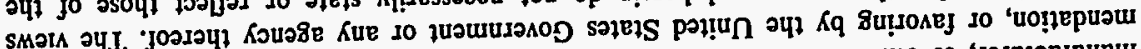

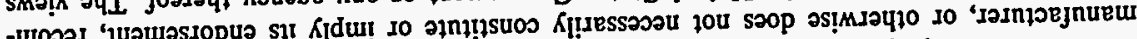

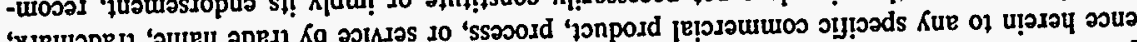

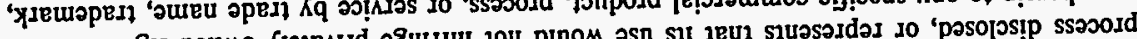

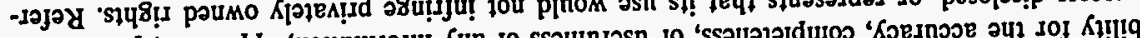
so 'jonposd 'snjesedde 'uo!̣eurroju! Kue jo ssaujnjasn

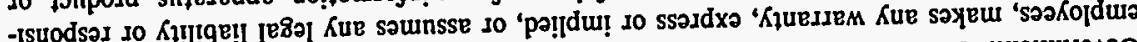

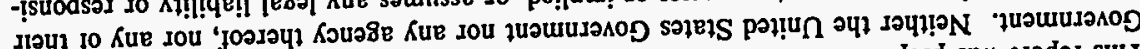
ją jo Kü

\section{\&มWIVTDSI}

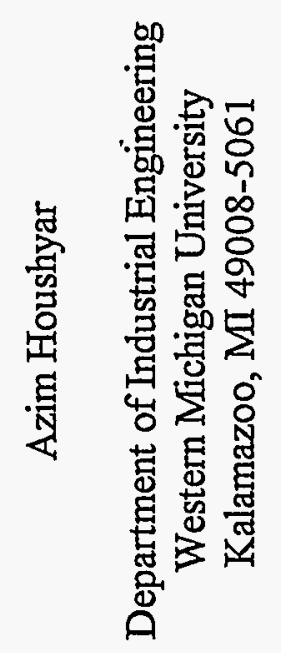

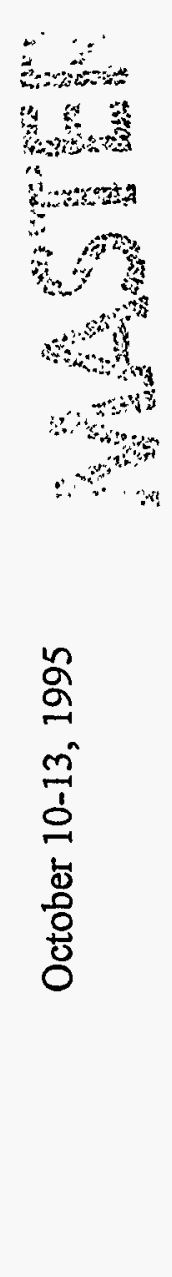

ఫृ

苨㤩 马े क 0.5 뗑응 空兵 . 舟四 田 次 $\varangle$ 臬 


\title{
Planning and Supervision of Reactor Defueling Using Discrete Event Techniques
}

\author{
Humberto E. Garcia \\ Azim Houshyar \\ George R. Imel \\ garcia@anl.gov, azim@anl.gov, imel@anl.gov \\ Argonne National Laboratory \\ P.O. Box 2528 \\ Idaho Falls, ID 83403-2528
}

\begin{abstract}
New fuel handling and conditioning activities for the defueling of the Experimental Breeder Reactor II are being performed at Argonne National Laboratory. Research is being conducted to investigate the use of discrete event simulation, analysis, and optimization techniques to plan, supervise, and perform these activities in such a way that productivity can be improved. The central idea is to characterize this defueling operation as a collection of interconnected serving cells, and then apply operational research techniques to identify appropriate planning schedules for given scenarios. In addition, a supervisory system is being developed to provide personnel with on-line information on the progress of fueling tasks and to suggest courses of action to accommodate changing operational conditions. This paper provides an introduction to the research in progress at ANL. In particular, it briefly describes the fuel handling configuration for reactor defueling at ANL, presenting the flow of material from the reactor grid to the interim storage location, and the expected contributions of this work. As an example of the studies being conducted for planning and supervision of fuel handling activities at ANL, an application of discrete event simulation techniques to evaluate different fuel cask transfer strategies is given at the end of the paper.
\end{abstract}

\section{Introduction}

The Experimental Breeder Reactor-II (EBR-II) is a $62.5 \mathrm{MW}$ (th) liquid metal reactor operated by Argonne National Laboratory for The United States Department of Energy. The reactor is located near Idaho Falls, Idaho at the Argonne-West site (ANL-W). Full power operation was achieved in 1964; the reactor operated continuously since that time until October 1994. In the summer of 1994, EBR-II was shut down and defueling was started. To assist in the planning for resources needed for this defueling campaign, a limited mathematical model of the fuel handling sequence was developed utilizing the appropriate reliability factors and inherent time constraints of each stage of the process [1]. This initial model is being refined to better predict transfer rates under different scenarios. The model will also facilitate planning of maintenance activities, as well as optimization of resources regarding manpower and modification effort. The central idea is to characterize this defueling operation as a collection of interconnected serving cells, and then apply operational research techniques to identify appropriate planning schedules for given scenarios. In addition, a supervisory system is being 
developed to provide personnel with on-line information on the progress of specified fueling tasks and to suggest courses of actions to accommodate changing operational conditions.

To meet the objective of the fuel (blankets and drivers) unloading program (complete unloading in two and an haft years), it was required that the normal fuel handling rate be accelerated. Blanket subassemblies (S/As) will be unloaded first over a period of about two years, followed by core driver S/As in 1997. Removal of blankets first will minimize exposure to operations crews, as blankets have the lowest decay power. Additionally, most core drivers require active cooling for periods of up to a year. As there are a limited number of cooled storage locations, unloading drivers first would cause a restriction to the overall flow similar to a capacitance effect. The inventory as of October 1, 1994 in the primary tank of fueled S/As (including blankets) that must be transferred is: 103 driver S/As (including control rods), 28 experimental S/As, and 321 depleted uranium blanket S/As for a total of 452 S/As to be removed. The schedule requires that these S/As be removed in two and a half year, which necessitates a rate of 14 transfers per month, or 167 per year. This rate is at least $50 \%$ greater than any historical experience, and is a factor of two above normal rates. The inventory, as of the writing of this paper (May 1995), is: 78 driver S/As (including control rods), 14 experimental S/As, and 252 depleted uranium blanket S/As for a total of 344 S/As to be removed. Thus, the required rate to the end of the program (March 31, 1997) is 188 per year or 16 transfers per month. The modeling approach described in this paper has assisted operations to determine the feasibility of achieving the required rate or the modifications on equipment and procedures required to achieve it, and has provided management with decision making information on a real time basis.

The path of spent fuel out of EBR-II to storage is a multistage process, involving complex operations at a minimum of four different facilities at the ANL-W site. Each of the facilities has several components necessary for fuel handling, which are described in more detail later in the paper. As a brief overview, the process is shown schematically in Figure 1. Specifically, the path to temporary storage in the Radioactive Scrap and Waste Storage Facility (RSWF) (where S/As are stored awaiting future conditioning) consists of five stages: (1) transfer from the reactor grid to the in tank storage basket, (2) transfer from the storage basket to the wash station (to wash residual sodium from the S/A) using the Fuel Unloading Machine (FUM) to load an S/A into the Inter-Building Cask (IBC), (3) transfer from the wash station via the $\mathrm{IBC}$ to the Hot Fuel Examination Facility (HFEF) where most S/As are placed in pits that are actively cooled, (4) transfer from the cooling pits to air storage racks (passively cooled), and (5) disassembly and packaging in HFEF for interim storage at RSWF in pits. As indicated in Section 4.C, spent S/As are then retrieved from RSWF and conditioned at the Fuel Cycle Facility (FCF) not shown in Figure 1. Each stage has a set of criteria that must be satisfied before a subassembly can be received. The criteria are based on decay power and age of the S/A: decay power to ensure adequate cooling will be available, either active or passive, and age to ensure that certain isotopes have decayed to minimize the source term in facilities outside the reactor. Each stage typically has complicated handling and/or cooling equipment that must be periodically maintained, leading to both planned and unplanned downtime. 


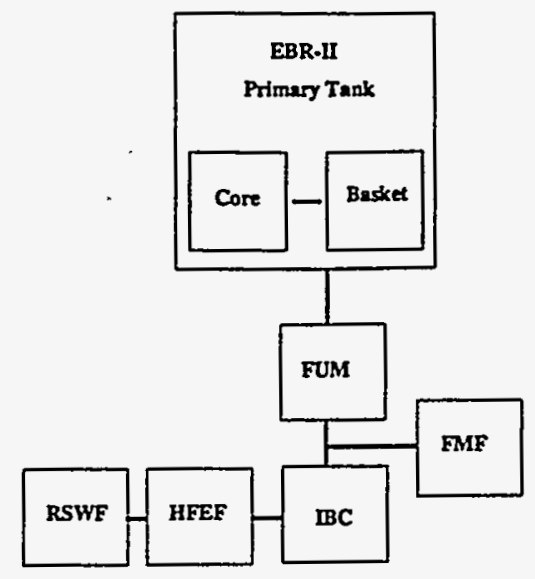

Figure 1. Fuel Handling at ANL-W.

The ANL-West site is equipped with resources and facilities suitable for handling, examining, characterizing, and conditioning spent fuel. Some of these fuel operations involve simple tasks that are accomplished following single operational procedures. Other tasks are more complex involving diverse resources and completed under several operational possibilities. To model these processes, the ANL-W resources can be characterized as an ensemble constructed of manifold cells of limited resources. These resources must function together in a safe, productive and a cost-effective manner. Although there has been considerable success in improving the rate of fuel transfers and conditioning at ANL-W, limited formal studies have been conducted to suggest better ways to utilize the existing resources. However, the ability to simulate diverse operational situations and thus device strategies to make the entire system run more efficiently has been identified to be a useful planning tool for current and future plant designs. In particular, it could be productive to have a supervisory system in plant to monitor process progress and provide advisory information to managers and operators when scheduling fuel handling and conditioning tasks. This research work will describe the use of discrete event simulation, analysis, and optimization techniques to plan, supervise, and perform the fuel unloading tasks in a way that will improve safety, productivity, and cost reduction. A brief introduction to the fuel handling capabilities at ANL-W is given next.

\section{Description of Material Flow}

Five types of S/A are involved during the current defueling operations at ANL-W: blankets, drivers, experiments, safety and control rods. It is the objective of the defueling task to remove all irradiated S/A out from the reactor grid, and prepare them for interim storage prior to conditioning. This fuel conditioning will be done at the Fuel Cycle Facility (FCF) as later presented in Section 4.C. In addition, it is required that any irradiated S/A removed from the reactor grid be replaced with a dummy S/A in order that proper spacing in the grid be maintained. This operation should be performed in such a way to minimize the time required to get all irradiated S/A out from the reactor grid. To achieve this objective, the following defueling strategy is executed. The flow of material for reactor defueling and fuel conditioning is shown in Figure 2. At FMF, new dummy S/As are manufactured. These S/As are transported to EBR-II using the Subassembly Carrier (SAC) via a pickup truck. Spent irradiated S/As are removed from the EBR-II reactor using the Inter-Building Cask (IBC). The reactor is loaded with stainless steel S/As. Spent S/As are transported to the Wash/Dry Station 
at FCF via a crane/cart arrangement. After washing the S/A to remove residual sodium, the IBC containing the S/A is sent either to HFEF via a cask truck or to the Fuel Conditioning Station also at FCF via a cart. After a certain cooling period at HFEF, which depends on the type and irradiation history of the S/A, S/A are disassembled and the contents placed in the Hot Fuel Examination Facility 5 cask $(H F E F-5)$ and transported to RSWF via the big rig. Stored S/A will be then retrieved from RSWF and sent to the Fuel Conditioning Station at FCF for final conditioning. Notice then that to move S/A between these facilities, specialized containers are utilized. In particular, the SAC is used to move new S/A, an IBC (two available) to move irradiated S/A and the HFEF-5 cask to move pre-conditioned S/A. Similarly, to move these containers, several transporting mediums are utilized. In particular, a pickup truck to move the SAC, a crane/cart to move a given IBC between EBR-II and FCF, the cask truck to move an IBC between FCF and HFEF and a rig to move the HFEF-5 between HFEF and RSWF.

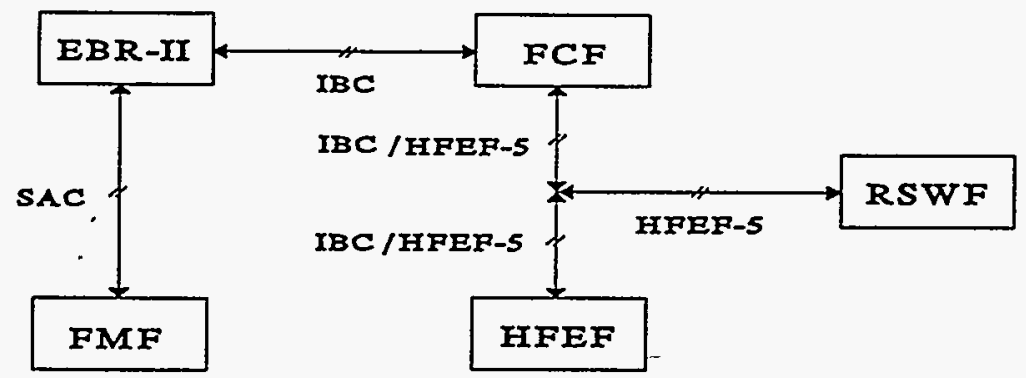

Figure 2. Flow of material during reactor defueling.

Assuming that there are sufficient new S/As at EBR-II, the flow of material for reactor defueling is as follows. A replacement dummy S/A is first introduced into EBR-II and then an irradiated S/A is removed in an IBC and shipped to FCF for washing. This loaded IBC is subsequently taken either to HFEF or to the Fuel Conditioning Station (FCS) at FCF where the irradiated S/A is retrieved from the IBC. While the particular IBC is taken back to the Wash/Dry Station at FCF for drying before returned to EBR-II, the just received S/A is conditioned either at HFEF or at FCF. In particular, at FCF, irradiated S/A are further conditioned at the Fuel Conditioning Station. On the other hand, at HFEF, S/As are stored in either cooling pits or air storage racks (depending on decay heat) before being disassembled and shipped to RSWF using the HFEF-5 cask. After emptying the HFEF-5 at RSWF, this cask is returned to HFEF. When thus determined, S/A will be then retrieved from RSWF and shipped to FCF for further conditioning. This completes an S/A disposal cycle.

\section{Research Contributions to Improve the Defueling Task}

Four main contributions will arise from this research in relation to the defueling task. First, a discrete event model of the complete defueling operation is being developed. The model has enough details to match the actual operations. Second, discrete event analysis techniques are being utilized on this model to characterize and predict the behavior of the actual process. In principle, fuel handling and conditioning can be achieved without carefully analyzing it under formal techniques such as those utilized in manufacturing engineering. However, feasible operational states and changes may be far more difficult to categorize, correctly interpret, and respond whenever executing defueling tasks. To this end, simulation models can reveal undesirable system characteristics and production problems, such as bottlenecks, before they appear on the actual process. In addition, several discrete event techniques, such as perturbation analysis, can be incorporated in current operations to improve 
the defueling tasks. Third, a supervisory system is being developed to assist defueling operations. A suggested architecture for its operation is given in Figure 3.

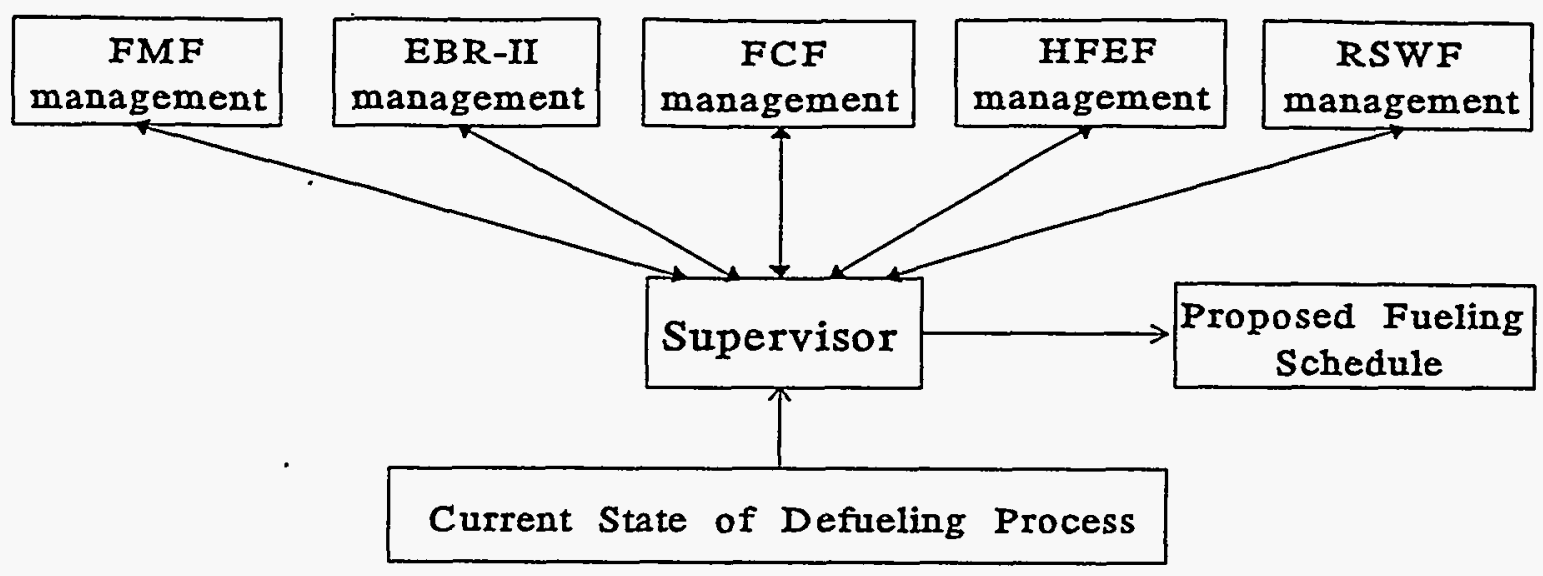

Figure 3. Supervisory architecture.

The supervisory system will suggest scheduling activities so that the complete defueling operation can be executed more safely, more economically and more efficiently. In addition, the supervisory agent is expected to substantially improve flexibility and safety by alleviating operators of routine and complex planning tasks and providing timely information for decision making. In particular, this supervisory agent will be most valuable for scheduling maintenance from different facilities. In this respect, the supervisor will schedule equipment maintenance at each facility involved in the defueling task in such a way that these operations will minimally perturb the defueling task. In addition, the supervisor will maintain a consistent central database so that operational requirements originated from each facility can be all scheduled in a plant-wide operation strategy that is acceptable for all facilities and adequate for the defueling task. This will greatly improve communication at the managerial level between different facilities. To implement this supervisory entity, discrete event optimization techniques, such as Rapid Learning, will be investigated. Finally, the introduction in the nuclear industry of some of the analysis and synthesis techniques provided by the discrete event theory is expected to be an important contribution of this research.

\section{Specific Facility Operations for Defueling}

\section{A Fuel Manufacturing Facility (FMF)}

This facility generates new S/A. When EBR-II was operational, the S/As were fueled, but now they are simply stainless steel dummies that provide the correct grid spacing for the fuel handling equipment. The facility can be characterized as a source for new S/A with a high productive capacity (i.e., it is not expected to be a critical path component to the defueling process). Figure 4 shows the main resources and materials produced at this facility.

\section{B Experimental Breeder Reactor II (EBR-II)}

In relation to the defueling task, this facility consumes new S/A and generates irradiated $\mathrm{S} / \mathrm{A}$. It can be characterized as the queue shown in Figure 5. In particular, new S/A enter Buffer 1 (the EBR-II reactor building) at discrete intervals. These S/As are then moved to 
FMF

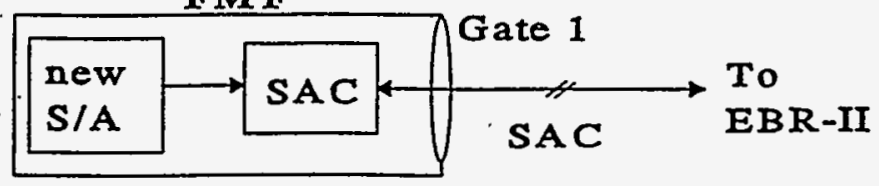

Figure 4. Defueling operations at FMF.

Buffer 2 of finite capacity (the storage basket submerged in the reactor tank). New S/As are then processed by Server 1 (the reactor grid), which processes $\mathrm{N}$ elements simultaneously. Irradiated spent S/A are then stored in Buffer 2. Finally, irradiated S/A leave this queue.

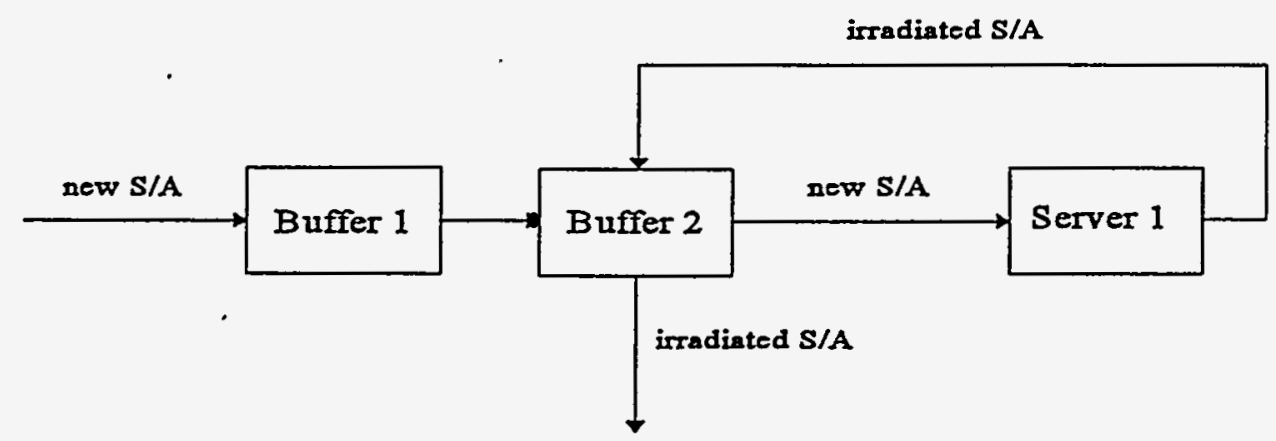

Figure 5. Equivalent queuing representation for defueling operations at EBR-II.

To relate this queue with physical resources, Buffer 1 corresponds to the New S/A Rack, Buffer 2 to the Storage Basket, and Server 1 to the EBR-II nuclear reactor grid as shown in Figure 6. The IBC and Auxiliary Coffin (AC) are devices where S/As need to be placed so that they can be retrieved from or introduced into the reactor tank, respectively. That is, to remove an irradiated S/A from the reactor tank, an IBC must be first placed at Port 1 where the Fuel Unloading Machine (FUM) can load the S/A into it. Similarly, to introduce a new S/A into the reactor, the AC is first loaded with the new S/A; the AC is then placed at Port 1 where the FUM can retrieve the new S/A and introduce it in the reactor. On the other hand, the Reactor Transfer Mechanism (RTM) and the transfer arm are handling S/A equipments utilized to move S/A between the reactor grid, the storage basket, and the FUM as seen in Figure 6.

When moving S/A in and out of the reactor tank, two fuel handling operations are defined, namely, unrestricted fuel handling (UFH) and restricted fuel handling (RFH). During UFH operations, S/A can be transferred between the reactor grid and the storage basket. This operation then involves the reactor grid, the RTM, the transfer arm and the storage basket, as shown in Figure 6. On the other hand, during RFH operations, S/A can be transferred between the storage basket and the AC or IBC. This operation then involves the storage basket, the transfer arm, the FUM and either the AC or a given IBC, as shown in Figure 6. In summary, UFH transfers involve S/A movements without leaving the reactor tank while RFH transfers involve S/A movement in and out of this tank. It is important to indicate here that UFH operations impose restrictive conditions that the reactor must satisfy to meet safety considerations (because transfers are occurring in or out of the reactor grid). On the other hand, RFH operations can be executed almost at any time. This implies that when the reactor is in UFH mode, some RFH transfers (i.e., between the basket and Port 1) would be allowed to occur. However, transfers of each type cannot occur simultaneously because they both require the transfer arm during S/A movements, as seen in Figure 6. In addition under this 
mode, it is possible (for certain S/A positions) to move S/A directly between the reactor grid and Port 1 bypassing the storage basket. The scheduling of RFH operations while in UFH mode could be considered when planning fuel handling. This is feasible because the time required to complete a UFH transfer is much shorter than the time to complete an RFH operation, which includes moving S/A out from the reactor tank, placing it in an IBC, moving it out of EBR-II and placing another IBC at Port 1. On the contrary, no UFH transfer (i.e., between the reactor grid and the storage basket) can occur whenever the reactor is in RFH mode.

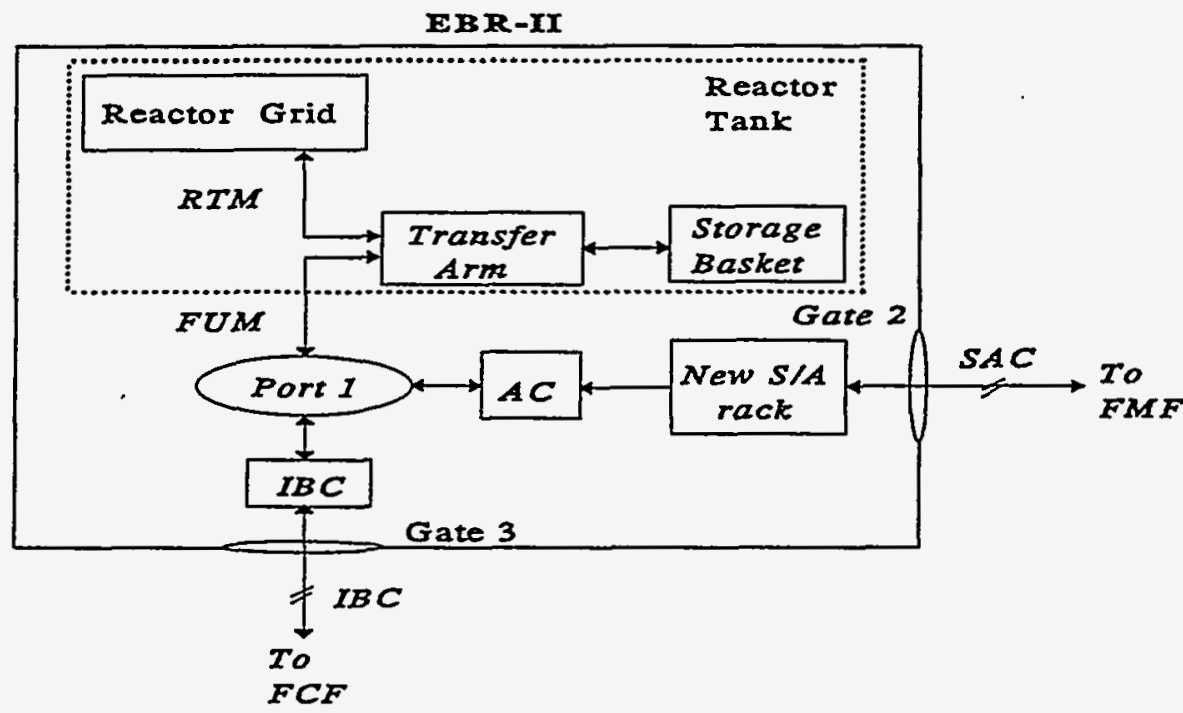

Figure 6. Defueling operations at EBR-II.

\section{C Fuel Cycle Facility (FCF)}

In relation to the defueling task, this facility executes three operations. It can be characterized as composed of five servers, with operational functionality of the first two conditioned to the current state of each other. This operational lock between Server 2 and Server 3 is represented in Figure 7 by a line with circle connecting them. The operations executed are washing an IBC, drying an IBC, or fuel conditioning. During washing, an IBC is internally washed to remove any residual sodium on the S/A. During drying, an IBC is dried to eliminate any humidity so it can be loaded with another S/A. The drying operation takes about twice as much time as it takes to wash. During fuel conditioning, the fuel elements of a driver or a blanket are conditioned to comply with fuel disposal guidelines. Physically, three stations exist at FCF where washing, drying, or fuel conditioning can be executed, as illustrated in Figure 8. However, an operational lock between Station 1 and Station 2 results from a design constraint that permits only one of these stations to be performing washing at any given time. That is, under maximal use, it is only possible to have either Station 1 and Station 2 simultaneously drying or one station drying and the other washing. Similarly, there exist restrictions for moving IBCs inside of FCF. For example considering Station 1 and Station 2, assume that an IBC has been loaded with an S/A at EBR-II and taken to Station 1 to be washed. In addition, assume that the other IBC was unloaded at HFEF (to remove its S/A) and has now been taken to Station 2 to be dried. The states to follow this situation is that the IBC at Station 1 will be taken to HFEF while the IBC at Station 2 will be moved to EBR-II. In the case that both stations end their services at the same time, it could be desirable to move both 


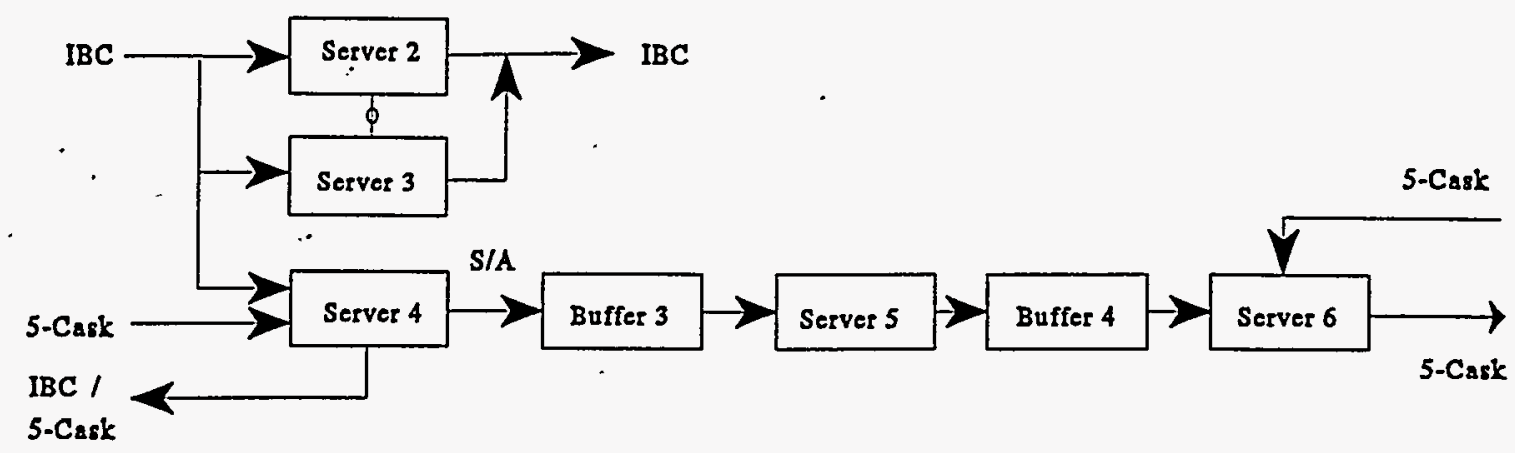

Figure 7. Queuing representation for defueling operations at FCF.

IBCs out from FCF at once. However, this cannot be done simultaneously because they need to share common exit paths, as shown in Figure 8. For this case, a solution could be to move the IBC at Station 1 out to HFEF through Gate 5 and then move the IBC at Station 2 to EBRII through Gate 4.

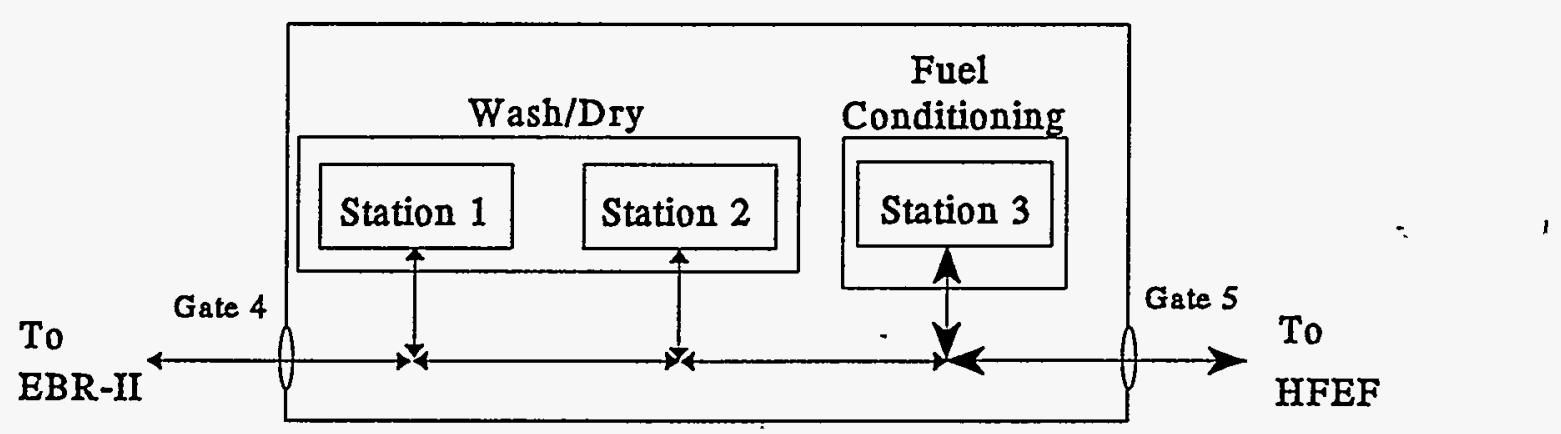

Figure 8. Defueling operations at FCF.

\section{D Hot Fuel Examination Facility (HFEF)}

In relation to the defueling task, this facility consumes irradiated S/A and generates both waste material (e.g., hardware) and spent fuel elements packaged for interim disposal at RSWF. In brief, a fuel cycle at HFEF starts with unloading the $\mathrm{IBC}$ at the corresponding port and removing the S/A it carries. The S/A may or may not be stored for some given time (which can be up to a year or more) in several storage areas. Then, an S/A is dismantled and its different byproducts stored in different waste cans. Finally, the HFEF- 5 cask is placed at the corresponding port and a filled waste container is loaded into it in order to be transported to RSWF. A queuing representation of the activities executed at HFEF is given in Figure 9 with a discussion on the flow of materials and different resources used at HFEF to be given next.

During fuel handling at HFEF, several types of repositories are utilized. They can be broadly classified into two types, namely, baskets and inner containers (IC). Baskets are used to hold together several items of a given type, with these items resulting from processing S/A. Three types of baskets are mentioned here, namely, Blanket Storage Baskets $(B S B)$, Fuel Storage Baskets for Mark-II fuel (FSB-2), and Fuel Storage Baskets for Mark-III fuel (FSB-3). A basket may require the conditioning of several S/As before it is full. For example, up to six S/A blankets, up to three S/As with Mark-II fuel elements and up to two S/As with Mark-III 
fuel elements can be held in a BSB, an FSB-2, and FSB-3, respectively. Subsequently, a specified number of baskets of a given type are used to fill an inner container. Three types of inner containers are mentioned here, namely, the Blanket Storage Containers (BSC), Short North (SN) and Fuel Storage Containers (FSC). A container may require one or more baskets of a given type before it is full. For example, one BSB is held in a single BSC while up to two FSBs (i.e., FSB-2 or FSB-3) can be held in an FSC. As discussed later, a given SN is directly filled with irradiated hardware (without the need of a basket) resulting from conditioning a variable number of S/A.

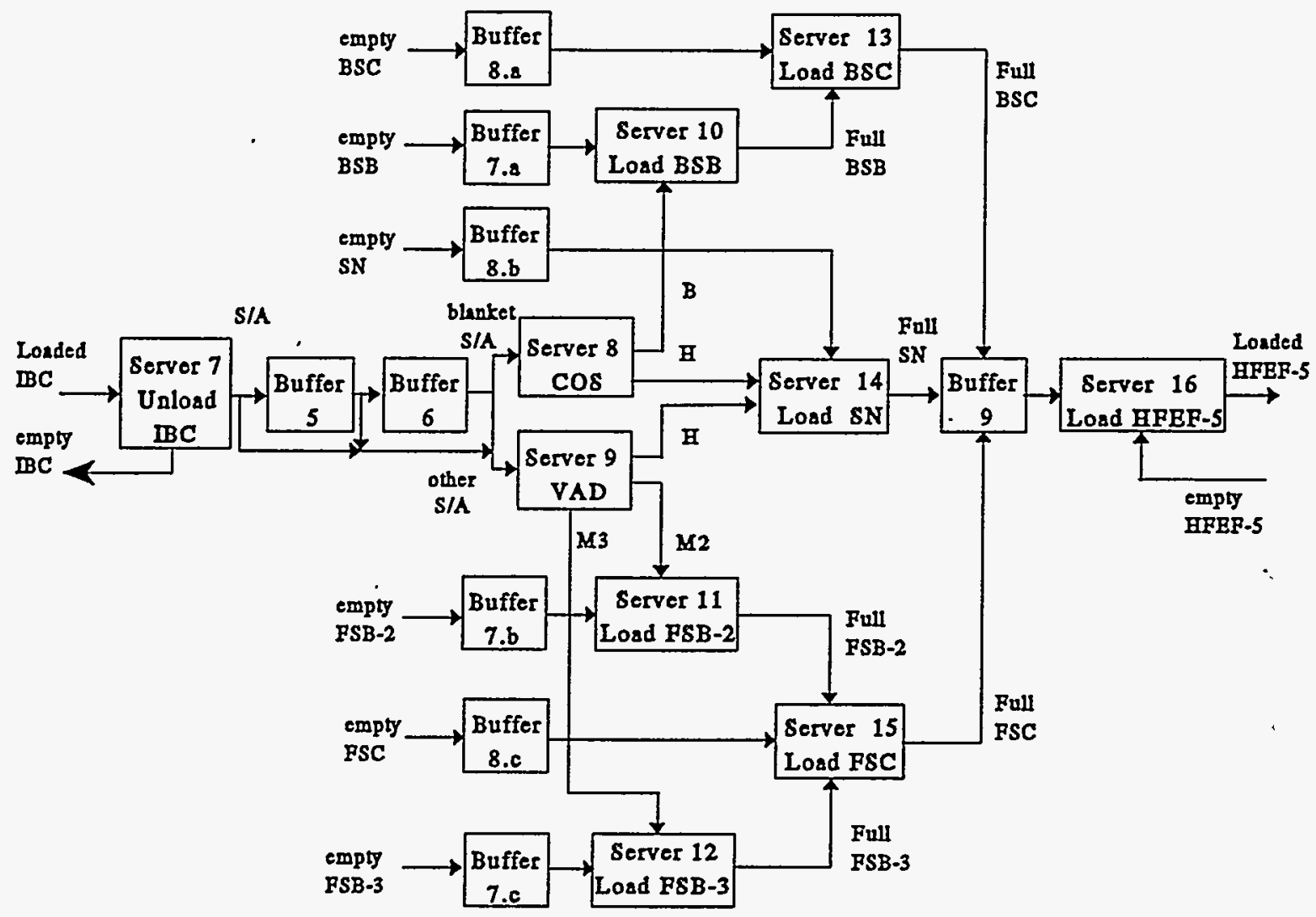

Figure 9. Queuing representation for defueling operations at HFEF.

Similarly, several types of processing are executed at HFEF. They can be broadly classified into three, namely, loading of a repository, unloading of a repository and dismantling an S/A. Two unloading operations are executed at HFEF. One is executed by Server 7 which removes an S/A carried by the given IBC at the corresponding unloading port. The other unloading operation, not shown in Figure 9, consists of removing an empty IC from the HFEF5 cask. This operation will be further discussed later. Several loading operations are executed at HFEF where a given repository is filled. For example in Figure 9, Server 11 takes an empty FSB and loads it with items M2 (to be defined later) generated by Server 9 . Similarly, Server 16 takes one inner container (e.g., an BSC, an SN, or an FSC) and loads it into the (empty) HFEF-5 cask. Dismantling operations break an S/A down into several items. In particular, two servers (i.e., Servers 8 and 9) execute dismantling tasks at HFEF as seen in Figure 9. Server 8 and Server 9 correspond to the Cutoff Saw (COS) and the Vertical Assembler Dismantler (VAD) devices, respectively. At the COS, the top end fixture and lower adapter of a blanket S/A are cut to reduce the S/A size. This operation generates two types of items, namely, Hardware $(\mathrm{H})$ and chopped Blankets $(\mathrm{B})$. $\mathrm{H}$ items are irradiated metallic parts resulting from 
the S/A while B items are blanket S/A without handling fixtures and lower adapters. At the $V A D$, spent fuel elements are removed from an S/A (other than blankets) by separating the fuel elements from the hardware comprising the S/A. This operation generates two types of items, namely, Hardware $(\mathrm{H})$ or fuel elements, which can be Mark-II fuel elements (M2) or Mark-III fuel elements (M3). Again, $\mathrm{H}$ items are metallic parts resulting from this dismantling process.

To provide fuel conditioning capability, HFEF is essentially a hot cell. In particular, this hot cell consists of two cells, namely, the Decon and Main cells, as seen in Figure 10.

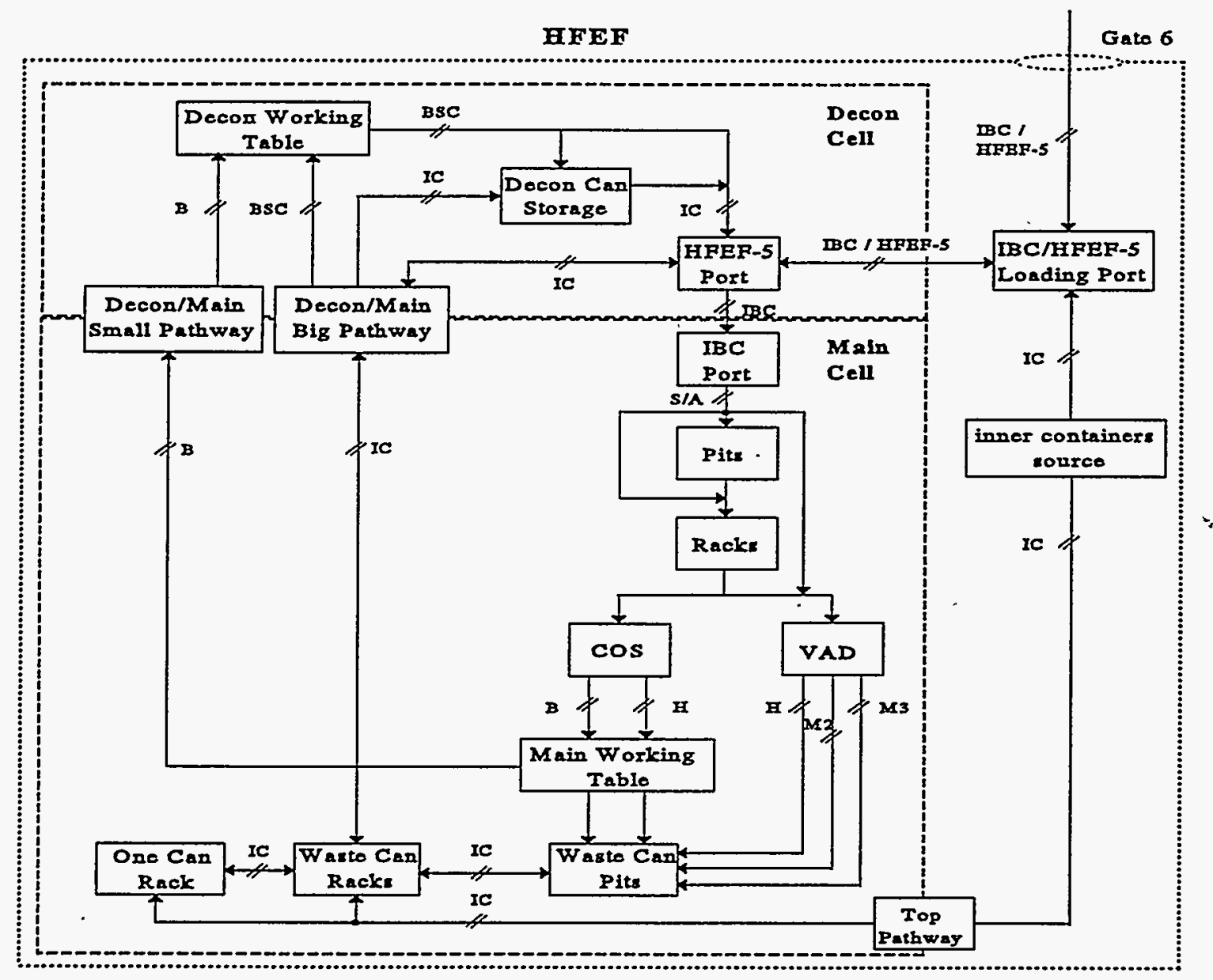

Figure 10. Defueling operations at HFEF.

While the Decon cell is an air-atmosphere hot cell, the main cell is an inert-gas (argon) atmosphere hot cell. In each of these cells, there exists several resources specialized for fuel handling and conditioning. First, there exist storage areas for irradiated material that are distributed between the Decon and Main cells. In particular, pits are storage locations for S/As that are used to cool S/A with a forced argon cooling system. There are 10 pits, each one with the capacity to hold up to 4 S/A; only five pits can hold control rods S/A in addition to the other S/A types. Racks are storage locations for S/A that are used to cool S/A by natural convection. There are five racks, each one with the capacity to hold up to $10 \mathrm{~S} / \mathrm{A}$. In relation with Figure 9, Buffer 5 and Buffer 6 corresponds to both pits and racks. There are also storage locations for waste baskets (i.e., BSB, FSB-2, and FSB-3). In Figure 9, Buffers 7.a, 7.b and 7.c correspond to these locations distributed around the hot cell. Similarly, there are storage locations for waste inner containers (i.e., SN, BSC, and FSC). Buffers 8.a, 8.b and 8.c as well 
as Buffer 9 correspond to waste inner container storage locations situated in the Decon Working Table, Decon Can Storage, One Can Rack, Wastes Can Pits, and Waste Can Racks, as seen in Figure 10. Each of these areas can hold up to one, three, one, three, and two inner containers, respectively; however, to meet safety guidelines, the Decon Working Table, Decon Can Storage, Waste Can Pits, and Waste Can racks can each have no more than one non-empty $\mathrm{BSC}$ or FSC at once. Second, there is fuel handling equipment (e.g., carts, bridge cranes, electro-mechanical manipulators, periscopes and sealed master/slave manipulators) used for the unloading and loading operations of material and containers. These tasks are executed by the Servers 7, 10-16 of Figure 9 and at most require the intervention of an operator. Finally, there are the dismantling operations executed by the Cutoff Saw (Server 8) and the Vertical Assembler Dismantler (Server 9).

\section{E Radioactive Scrap and Waste Facility (RSWF)}

In relation to the defueling task, this facility provides interim storage of EBR-II spent fuel and waste hardware. The facility can be characterized as a sink with a large capacity for irradiated S/A. Figure 11 shows the main resources and materials stored at this facility.

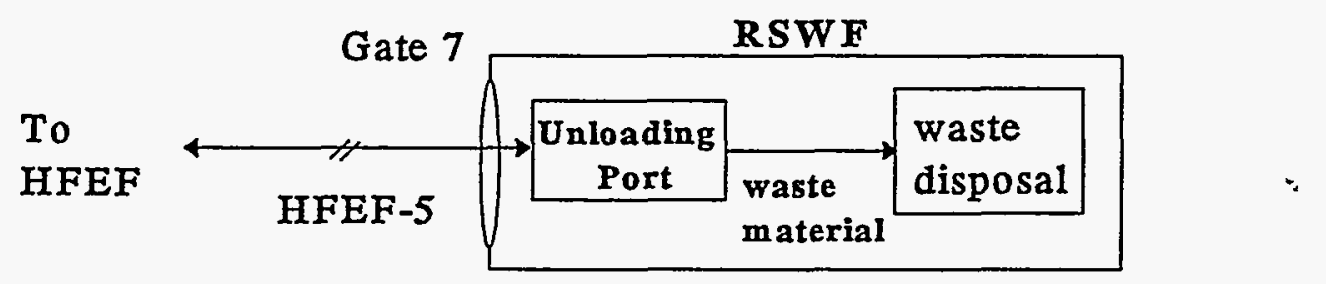

Figure 11. Defueling operations at RSWF.

\section{DES Techniques in Defueling Analysis and Designs}

Discrete Event techniques are being used in several tasks related with the defueling operations at ANL-W. These tasks include off-line system analysis and designs of current fuel handling operations as well as on on-line supervisory systems. To illustrate some of the outcomes obtained from these studies, an application of discrete event simulation is presented to analyze and recommend a cask transfer routing strategy among facilities. In particular, the problem was to determine which route would lead to less time and less cask transfers for completing reactor defueling. Two possible cask routing strategies $A$ and $B$ are defined in Table 1. For example, under Strategy $B$, the routing for blankets originated at HFEF (entry $[4,2])$ is to ship them from HFEF to RSWF; then, when possible, back to HFEF and finally to FCF. The number of S/As shipped in a given transfer between two facilities depend on several factors not discussed here. To compare these routing strategies, detailed discrete event models of each facility and of the cask transfer policies were developed. Simulating results under each routing scenario are shown in Table 2 and Figure 12. From Table 2, it can be seen that it takes 86 more cask transfers and 126 additional days to complete the defueling operation under Strategy B than under Strategy A. These results indicate that Strategy A is a preferable choice over Strategy B. However, Strategy A presumes that FCF can accept blankets previously treated at HFEF. If this is not the case, the corresponding cost involved in providing FCF with this capability must be waited against the benefits obtained in reducing cask transfers and completion time when identifying the optimal solution. 